\title{
Properties of Ga-Doped Magnetite Nanoparticles
}

\author{
K. Ré́Ko ${ }^{a, *}$, U. KlekotkA ${ }^{b}$, B. Kalska-SzostKo ${ }^{b}$, D. Soloviovi, ${ }^{c, e}$, D. SatuŁA ${ }^{a}$ \\ AND J. WALISZEWSKI ${ }^{a, c}$
}

${ }^{a}$ University of Bialystok, Faculty of Physics, 15-245 Białystok, ul. K. Ciołkowskiego 1L, Poland

${ }^{b}$ University of Bialystok, Institute of Chemistry, 15-245 Białystok, K. Ciołkowskiego 1K, Poland

${ }^{c}$ Frank Laboratory of Neutron Physics, Joint Institute for Nuclear Research, Joliot-Curie 6, 141980 Dubna, Moscow region, Russia

${ }^{d}$ Moscow Institute for Physics and Technology, Dolgoprudny, 141701, Russia

${ }^{e}$ Taras Shevchenko National University of Kyiv, Kyiv, 01033, Ukraine

\begin{abstract}
This paper reports our progress toward developing the best methods to prepare Ga-doped magnetite nanoparticles. Results of X-ray powder diffraction, small angle neutron scattering and the Mössbauer spectroscopy measurements of core-shell $\mathrm{Ga}_{0.2} \mathrm{Fe}_{2.8} \mathrm{O}_{4} / \mathrm{Fe}_{3} \mathrm{O}_{4}$ and $\mathrm{Fe}_{3} \mathrm{O}_{4} / \mathrm{Ga}_{0.2} \mathrm{Fe}_{2.8} \mathrm{O}_{4}$ nanoparticles are presented. They all were found to crystallize in the cubic structure. The diffraction data confirmed high sensitivity of the phase homogeneity depending on the preparation method. The volume of the unit cell has been proved to be independent of the gallium content. X-ray measurements disclosed that Ga atoms occupy preferentially (A) sites, while (B) sites are entirely occupied by Fe cations. The Mössbauer results are fully consistent with diffraction ones when $\mathrm{Ga}_{0.2} \mathrm{Fe}_{2.8} \mathrm{O}_{4}$ is a core of core-shell systems. The small angle neutron scattering was performed in order to test the shape and size of particles.
\end{abstract}

DOI: 10.12693/APhysPolA.134.998

PACS/topics: 61.05.fm, 61.05.fg, 61.05.Qr, 61.46.--w, 76.80.+y

\section{Introduction}

A great interest in magnetic nanomaterials is caused by their expected application. The important application target is cancer medicine, where nanomaterials can play various roles from the drug delivery media to structural material in biocomposites [1]. Huge attention has been paid to tumor treatment by hyperthermia $[2,3]$ with use of various types of magnetic particles. Particularly, iron tetroxide based nanoparticles with superior magnetic properties and properly surface functionalized are being intensively investigated to achieve highly efficient carcinogenic cell destruction through so-called therapeutic hyperthermia ${ }^{\dagger}$.

This paper concentrates on the ordered $\mathrm{Ga}_{0.2} \mathrm{Fe}_{2.8} \mathrm{O}_{4}$ nanoparticles that similarly to matrix $\mathrm{Fe}_{3} \mathrm{O}_{4}$ crystallize in Fd-3m structure (no. 227), shown in Fig. 1, and exhibit interesting properties of the selective location of Ga impurities. Due to the relatively small admixture of gallium, two extreme models of structural ordering in the matrix magnetite were tested. The powder patterns were initially modelled employing the spinel structure with $\mathrm{Ga}^{3+}$ ions of the smallest radius $(r=62 \mathrm{pm})$ occupy the (B) site. In the matrix the Wyckoff positions $16 \mathrm{~d}$ $\left(\frac{1}{2}, \frac{1}{2}, \frac{1}{2}\right)$ are occupied by $\mathrm{Fe}^{3+}(r=64.5 \mathrm{pm})$ and $\mathrm{Fe}^{2+}$

*corresponding author

†The therapeutic hyperthermia is a type of treatment in which body tissue is exposed to high temperatures to damage and kill cancer cells or to make cancer cells more sensitive to the effects of radiation and certain anticancer drugs (as defined by the USA National Cancer Institute).
( $r=78 \mathrm{pm})$ equally. Afterward, in the second model of structural ordering, gallium ions are located entirely at the Wyckoff $8 \mathrm{~b}\left(\frac{1}{8}, \frac{1}{8}, \frac{1}{8}\right)$ with the fixed oxygen occupation at the Wyckoff position 32e $(x, x, x)$, where the $x$-value is the only variable parameter to be determined. Significantly different quality of the refinements of the diffraction patterns allowed a free analysis of the (A)-(B) type of gallium disordering.

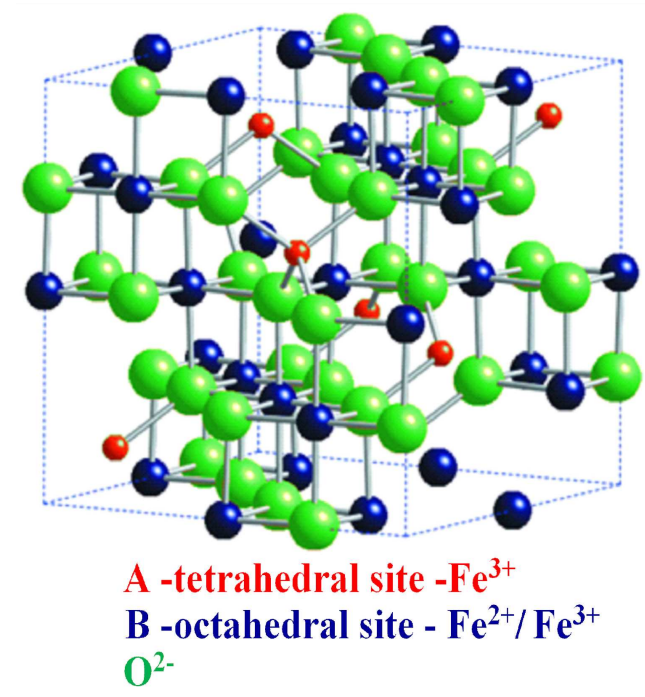

Fig. 1. The unit cell of the $\mathrm{Fe}_{3} \mathrm{O}_{4}$ cubic inverse spineltype structure.

Ferrimagnetism of the magnetite below the Curie temperature $T_{\mathrm{c}} \approx 850 \mathrm{~K}$ is created by ferromagnetic moments of ferric species on tetrahedral sites, and antiferro- 
magnetic moments on octahedral sites, occupied by ferric and ferrous species [4]. At $T_{\mathrm{c}}$ point, the thermal fluctuations destroy the exchange-coupled spins thus in-sites alignment of iron magnetic moments. In consequence, net magnetization strives to zero and superparamagnetic behavior is revealed. Noteworthy, as particle size decreases, the amount of exchange-coupled spins resisting spontaneous magnetic reorientation decreases as well, tending toward superparamagnetic magnetization. Consequentially, decreasing magnetite particle size should demonstrate the enhanced paramagnetic behavior.

It is well known if superparamagnetic magnetism dominates at room temperature, the effective phase transition temperature must be lower. Moreover, as particle diameter decreases, surface effects will eventually affect saturation magnetization. For example, it was discovered that below $10 \mathrm{~nm}$, saturation magnetization suddenly reversed trend and began decreasing with decrease of particle size due to surface effects. Particularly, due to the hyperthermia treatment reason, the magnetic properties and thermal stability of undoped and doped ferrite nanoparticles are of crucial importance. Although, magnetite-based nanostructures can be relatively easy to be obtained by different synthetic procedures [5], it is not trivial to obtain nanoparticles with exactly the same well-defined shape, size, and active surface. In order to obtain a desired shape and size, the strict control of the reaction conditions, such as the synthesis time, temperature, type and concentration of the precursors and added surfactants, must be maintained [6]. The previously obtained single phase of $80-100 \mathrm{~nm} \mathrm{Ga}_{0.9} \mathrm{Fe}_{2.1} \mathrm{O}_{4}$ particles of inverse spinel symmetry had motivated us to test metal chlorides precursors similarly to sol-gel method [6].

Another important aspect is preventing nanoparticles agglomeration, that is easier to achieve in the coreshell system $\mathrm{Fe}_{3} \mathrm{O}_{4} / \mathrm{Ga}_{0.2} \mathrm{Fe}_{2.8} \mathrm{O}_{4}$. According to X-ray absorption spectroscopy and X-ray magnetic circular dichroism measurements carried out on $\left(\mathrm{Ga}_{x} \mathrm{Fe}_{1-x}\right)_{3} \mathrm{O}_{4}$ nanoparticles with $(x=0.05-0.33)$ gallium doping, the average Fe moment is observed to increase resulting in an overall increase in the total moment of the material [7]. In fact, an admixture of $\mathrm{Ga}^{3+}$ at $\mathrm{Fe}_{3} \mathrm{O}_{4}$ as the nonmagnetic ions located at the tetrahedral sites no longer partially cancel the magnetic moment of iron ions in the octahedral sites. The core-shell with the magnetite nanoparticles doped gallium as a shell should easily follow the alternating magnetic field. Moreover, we expect that the investigation of nanoparticles with increase of gallium content at $\mathrm{Ga}_{x} \mathrm{Fe}_{3-x} \mathrm{O}_{4}$ in the core and shell will enforce the shift of the temperature of blocking superparamagnetism towards higher temperatures.

\section{Sample preparation}

The preparation of magnetite nanoparticles has been described in the earlier paper [5]. The most essential difference in the two different methods of sample preparation used by us consists of the environment of the seeds on which the particles grow (see Fig. 2). The former method is based on the coprecipitation of iron(II) and iron(III) chlorides in tetrabutylammonium hydroxide (TBAOH) solution - hereinafter referred to as "ch". In the second one - hereinafter referred to as "acac" — the original core of ferrite is formed during thermal decomposition of iron(III) acetylacetone and gallium(III) acetylacetone salts in an alcohol, carboxylic acid, and amine as surfactants and high boiling solvent, for example, a phenyl ether solution. This is a low-temperature reaction that was conducted at $80^{\circ} \mathrm{C}$ under Ar atmosphere. The $\mathrm{Fe}_{3} \mathrm{O}_{4}$ nanoparticles obtained from the $\mathrm{Fe}(\mathrm{acac})_{3}$ complex were modified with a $\mathrm{Ga}_{x} \mathrm{Fe}_{3-x} \mathrm{O}_{4}$ shell and vice versa the $\mathrm{Ga}_{x} \mathrm{Fe}_{3-x} \mathrm{O}_{4}$ core was modified with a $\mathrm{Fe}_{3} \mathrm{O}_{4}$ shell.
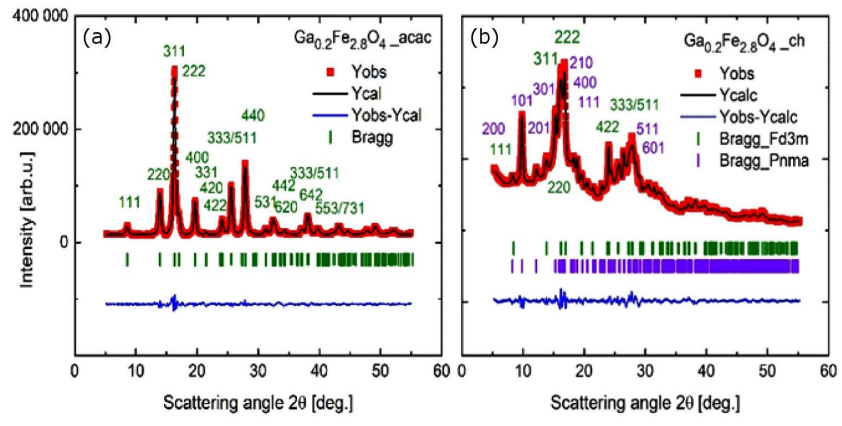

Fig. 2. RT X-ray powder diagrams experimental, calculated and the residual one for nominal $\mathrm{Ga}_{0.2} \mathrm{Fe}_{2.8} \mathrm{O}_{4}$ nanoparticles obtained by different methods.

\section{X-ray diffraction}

Room temperature XRD measurements were performed using Empyrean Panalytical powder diffractometer located at the Faculty of Physics University of Bialystok (FP, UB), with $40 \mathrm{kV}$ and $40 \mathrm{~mA}$ and Mo $K_{\alpha}$ radiation, $\lambda=0.7093187 \AA$ in the Bragg-Brentano geometry. A $2 \theta$ range from $5^{\circ}$ to $55^{\circ}$ was covered using a step of $0.026261^{\circ}$ and a count time of $3000 \mathrm{~s}$ per point. The diffraction patterns were analysed by the Rietveld-type profile refinement method using FullProf program [8]. For better readability, the goodness-of-fit parameters defined in Ref. [8] are also quoted.

These measurements have revealed the homogeneity of acac nanoparticles while the ch sample exhibits $54 \mathrm{wt} \%$ of the orthorhombic extra phase together with desirable inverse spinel structure. For better readability of the figures, some of the peaks shown have been left without indices.

The attempts of indexing the significant extra diffraction peaks in this sample succeed in indicating that the system under study is a goethite $\mathrm{FeO}(\mathrm{OH})$ phase that exhibits the orthorhombic Pnma space group and $a=9.866(4) \AA, b=3.006(1) \AA$ and $c=4.575(2) \AA$ lattice parameters. The refinements of $\mathrm{X}$-ray diagrams are shown in Fig. 2a,b. In spite of the high number of counts accumulated in peaks, the resolution functions of broadened lines are coupled with the microstrain coefficient 
and size distribution effect. The atomic scattering factors of iron and gallium, i.e. $f_{\mathrm{Fe}}$ and $f_{\mathrm{Ga}}$, easily distinguishable in X-ray diffraction experiments, allow recognizing the structural ordering (Eqs. (1a,b)) and in it in-site gallium ions concentration $x_{\mathrm{Ga}(\mathrm{A})}$ and $y_{\mathrm{Ga}(\mathrm{B})}$, respectively (Eqs. $(2 \mathrm{a}, \mathrm{b}))$. The analysis of the occupations of the $(\mathrm{A})$ and (B) sites allow to compare the estimated composition of the nanosystem with the nominal $\mathrm{Ga}_{x+y} \mathrm{Fe}_{3-x-y} \mathrm{O}_{4}$ one according to Eqs. (1a,b):

$$
\begin{gathered}
\xi_{\mathrm{Fe}(\mathrm{A})} f_{\mathrm{Fe}}=\frac{\left(2-x_{\mathrm{Ga}(\mathrm{A})}\right) f_{\mathrm{Fe}}+x_{\mathrm{Ga}(\mathrm{A}) f_{\mathrm{Ga}}}}{2}= \\
\left(1-\frac{x_{\mathrm{Ga}(\mathrm{A})}}{2}\right) f_{\mathrm{Fe}}+\frac{x_{\mathrm{Ga}(\mathrm{A})}}{2} f_{\mathrm{Ga}}
\end{gathered}
$$

and

$$
\xi_{\mathrm{Fe}(\mathrm{B})} f_{\mathrm{Fe}}=\left(1-y_{\mathrm{Ga}(\mathrm{B})}\right) f_{\mathrm{Fe}}+y_{\mathrm{Ga}(\mathrm{B})} f_{\mathrm{Ga}},
$$

where $\xi$ - in-site occupancy, if does not exceed one, is typical for pure magnetite part of core-shell particles. Due to all occupancies are normalized to unity, the appropriate gallium concentration at $\mathrm{A}$ and $\mathrm{B}$ positions can be calculated by the formulae

$$
x_{\mathrm{Ga}(\mathrm{A})}=\frac{52}{5}\left(\xi_{(\mathrm{A})}-1\right)
$$

and

$$
y_{\mathrm{Ga}(\mathrm{B})}=\frac{26}{5}\left(\xi_{(\mathrm{B})}-1\right) .
$$

According to the results presented at Table I the effective scattering amplitudes of the (B) sites were found to be close to iron atomic scattering factors, which means that gallium does not enter (B) positions. However, the occupancies of the (A) sites consistently disclose greater effective scattering amplitudes. The plausible explanation is the presence of gallium ions at the (A) positions. Such a model was discussed in detail in Ref. [7]. The model with the A-B disorder and untouched oxygen positions had also to be considered. All site occupancies were normalized to one. The value $x=0.253(5)$ obtained for $\mathrm{Fe}_{3} \mathrm{O}_{4}$ was used as an initial parameter. In order to reduce the free parameters during refinements for all measuring systems the isotropic Debye-Waller factor of value $B=0.566 \AA^{2}$ was fixed.

Results obtained by X-ray diffraction powder technique show that the non-shell core nanoparticles (see 3rd and 4th columns of Table I) as well as core-shell nanoparticles are single phase and ordered in the inverse spinel type structure (see 5th and 6th columns of Table I and Fig. 3a,b). This conclusion means that for the synthesis treatment applied here the estimated sample compositions do not differ from the nominal ones.

However, it is easy to observe much worse intensity to background ratio for nanoparticles where $\mathrm{Ga}_{0.2} \mathrm{Fe}_{2.8} \mathrm{O}_{4}$ is as a shell. The smaller particle size seems to be a reasonable explanation for this experimental fact. Another origin is higher organics content in the sample and in it better separation of the particles. The latter one promotes incoherent scattering. Noteworthy, the low angle scattering parts of diagrams up to 15 in the $2 \theta$ scale indicate the different contribution of organic surfactants.
TABLE I

Structural parameters for $\mathrm{Ga}_{0.2} \mathrm{Fe}_{2.8} \mathrm{O}_{4}$ as non-shell core and as core or shell of core-shell type nanoparticles: cubic lattice parameter - $a$, sites occupancies, position $(x, x$, $x$ ) of oxygen ions and goodness of fit (GOF) parameters obtained from RT X-ray measurements.

\begin{tabular}{l|l|c|c|c|c}
\hline \hline \multicolumn{2}{c|}{$\begin{array}{c}\text { Method } \\
\text { of preparation }\end{array}$} & $c h$ & acac & acac & acac \\
\hline \multicolumn{2}{c|}{$\begin{array}{c}\text { location } \\
\text { of } \mathrm{Ga}_{0.2} \mathrm{Fe}_{2.8} \mathrm{O}_{4}\end{array}$} & $\begin{array}{l}\text { core } \\
\text { non-shell }\end{array}$ & $\begin{array}{l}\text { core } \\
\text { non-shell }\end{array}$ & $\begin{array}{l}\text { core at } \\
\text { core-shell }\end{array}$ & $\begin{array}{l}\text { shell at } \\
\text { core-shell }\end{array}$ \\
\hline $\begin{array}{l}\text { lattice } \\
\text { parameter }\end{array}$ & $a[\AA]$ & $8.355(3)$ & $8.399(1)$ & $8.394(1)$ & $8.386(1)$ \\
\hline site & $\xi_{(\mathrm{A})}$ & $0.91(6)$ & $1.05(4)$ & $0.99(6)$ & $1.13(5)$ \\
occupancy & $\xi_{(\mathrm{B})}$ & $1.19(6)$ & $0.99(4)$ & $1.11(6)$ & $0.96(5)$ \\
& $\xi_{\mathrm{O}^{2-}}$ & $0.89(17)$ & $1.01(12)$ & $1.16(15)$ & $1.05(10)$ \\
\hline oxygen & & & & & \\
position & $x$ & $0.2432(9)$ & $0.2496(4)$ & $0.2509(6)$ & $0.2503(2)$ \\
parameter & & & & & \\
\hline \multirow{2}{*}{ GOF } & $R_{F}$ & 0.06 & 0.02 & 0.03 & 0.02 \\
& $R_{\mathrm{Bragg}}$ & 0.10 & 0.03 & 0.03 & 0.02 \\
& $\chi^{2}$ & 11.7 & 7.2 & 12.6 & 9.6
\end{tabular}
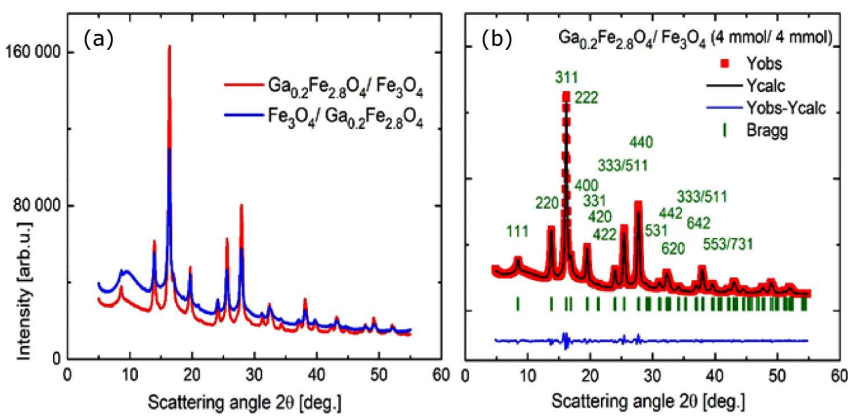

Fig. 3. RT X-ray diagrams of core-shell nanoparticles collected in identical statistical conditions (a) and the refinement of the $\mathrm{Ga}_{0.2} \mathrm{Fe}_{2.8} \mathrm{O}_{4} / \mathrm{Fe}_{3} \mathrm{O}_{4}$ particles (b).

Another important finding is observed rather weak dependence of the lattice parameter (see 3rd row of Table I) of the location of $\mathrm{Ga}_{0.2} \mathrm{Fe}_{2.8} \mathrm{O}_{4}$. Concerning biomedical applications in it the magnetic moment's interactions it seems to be extremely important to recognize the parameters of the unit cell as well as interionic distances dependences against of gallium content (paper in preparation).

\section{Small angle neutron scattering}

The SANS measurements were performed on YuMO spectrometer operating on IBR-2 (JINR, Dubna) $[9,10]$. SANS enables one to measure structural features by analysing the scattering pattern at very low angles from the direct neutron beam. By examining primary neutron beam, which is scattered at small angles, we can measure information that is directly proportional to the size and shape of nanometer-sized systems [6]. Spectrometer YuMO allows one to obtain $I(Q)$ curves in absolute scale where the typical SANS graphics represents the scatter- 
ing intensity $I$ as a function of the momentum transfer $Q=\left(\frac{4 \pi}{\lambda}\right) \sin (\theta)$ and depends on scattering angle $\theta$ and $\lambda$ - neutron's wavelength of the incident beam.

The spectrometer is characterized by relatively short data acquisition time which depends on the type of our samples and varies from $10 \mathrm{~min}$ to half an hour. All samples were measured at four temperature points in the range $20-100^{\circ} \mathrm{C}$. The particles' shapes, sizes, polydispersion coefficients, and morphologies were analysed using the SAS-view program [11].

In the simplest case of isotropic systems composed of isolated and identical particles embedded in a matrix with a constant neutron-scattering length density sld $\mathrm{ABO}_{4}=\frac{8\left(b_{\mathrm{A}}+b_{\mathrm{B}}+4 b_{\mathrm{O}}\right) \times 10^{11}}{V_{\text {unit } \operatorname{cell}\left[\AA^{3}\right]}} \mathrm{cm}^{-2}$ the intensities are proportional to the square of the form factor $F(Q)$ according to relations: $I(Q) \propto\left\langle|F(Q)|^{2}\right\rangle$, where the $b_{\mathrm{A}}, b_{\mathrm{B}}$, $b_{\mathrm{O}}$ are the appropriate neutron scattering lengths of $\mathrm{A}, \mathrm{B}$ and $\mathrm{O}$ sample elements at the above presented example.

The best model adopted for the description of $\mathrm{Fe}_{3} \mathrm{O}_{4} / \mathrm{Ga}_{0.2} \mathrm{Fe}_{2.8} \mathrm{O}_{4}$ and $\mathrm{Ga}_{0.2} \mathrm{Fe}_{2.8} \mathrm{O}_{4} / \mathrm{Fe}_{3} \mathrm{O}_{4}$ nanoparticles require the $F(Q)[12]$ described by volume of the whole particle $-V_{p}$, volume of the core $-V_{c}$, effective radius of the particles $r_{p}=$ core radius $\left(r_{c}\right)+$ thickness $(t h)$, and $s l d_{c}$ of core, sld $d_{s h}$ of shell and $s l d_{s}$ of solvent. The results of average values of $r_{c}=72.05 \pm 0.07 \AA$ and th $=13.95 \pm 0.07 \AA$, and $r_{p}=85.75 \pm 0.06 \AA$, disclose the sample where $\mathrm{Ga}_{0.2} \mathrm{Fe}_{2.8} \mathrm{O}_{4}$ is as a core of $\approx 18 \mathrm{~nm}$ of the effective diameter of spherical nanoparticles. The spherical core-shell model seems to be more adequate for $\mathrm{Ga}_{0.2} \mathrm{Fe}_{2.8} \mathrm{O}_{4} / \mathrm{Fe}_{3} \mathrm{O}_{4}$ nanoparticles, too. In this case, finally obtained particle's average parameters are as follows: $r_{c}=80.10 \pm 0.06 \AA$ and $t h=22.18 \pm 0.07 \AA$ and $r_{p}=102.35 \pm 0.06 \AA$.

The sample where $\mathrm{Ga}_{0.2} \mathrm{Fe}_{2.8} \mathrm{O}_{4}$ is as a shell indicate $\approx$ $20 \mathrm{~nm}$ of the effective diameter of spherical nanoparticles.

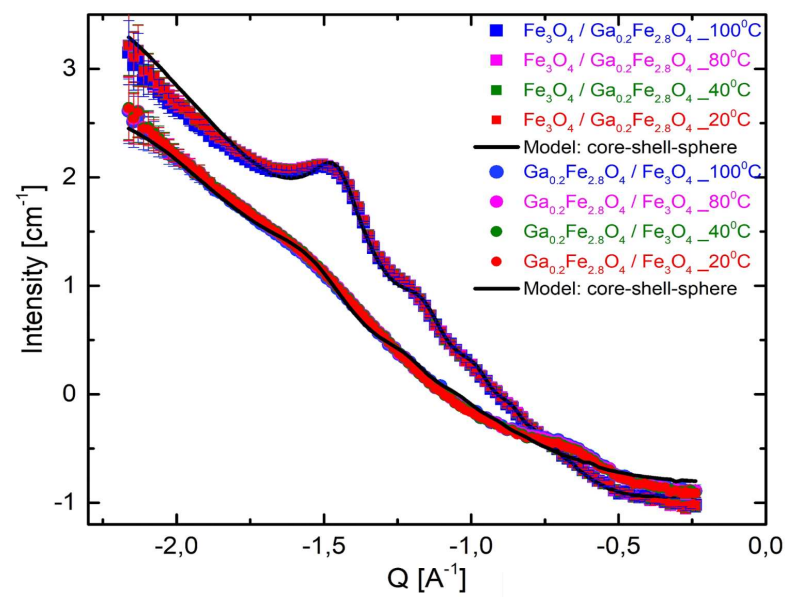

Fig. 4. SANS data collected at the temperature range up to $100^{\circ} \mathrm{C}$ for core-shell nanoparticles. The panel illustrates experimental curves in logarithmic scale (colored squares and circles) and the best fits (black solid lines) for spherical $\mathrm{Fe}_{3} \mathrm{O}_{4} / \mathrm{Ga}_{0.2} \mathrm{Fe}_{2.8} \mathrm{O}_{4}$ and $\mathrm{Ga}_{0.2} \mathrm{Fe}_{2.8} \mathrm{O}_{4} / \mathrm{Fe}_{3} \mathrm{O}_{4}$ one's particles.
The nanoparticles behaviour against temperature disclosed wide thermal stability of all measured core-shell systems (see Fig. 4). According to mentioned above shape models the average parameters still require confirmation by other less organic surfactants sensitive techniques.

\section{Mössbauer spectroscopy}

The room temperature (RT) Mössbauer spectra were measured with use of the standard spectrometer working in constant acceleration mode with a ${ }^{57} \mathrm{Co} \underline{R h}$ radioactive source. Metallic iron foil $(\alpha-\mathrm{Fe})$ was used as reference material. All samples were measured in the transmission mode. Measured RT Mössbauer spectra of core-shell nanoparticles $\mathrm{Fe}_{3} \mathrm{O}_{4} / \mathrm{Ga}_{0.2} \mathrm{Fe}_{2.8} \mathrm{O}_{4}$ and $\mathrm{Ga}_{0.2} \mathrm{Fe}_{2.8} \mathrm{O}_{4} / \mathrm{Fe}_{3} \mathrm{O}_{4}$ show in general superposition of superparamagnetic doublet in the central part of spectra, broad background sextet, and three relatively sharp sextets. Two of them with highest hyperfine magnetic field relates to magnetite $\mathrm{Fe}(\mathrm{A})$ (tetrahedral) and $\mathrm{Fe}(\mathrm{B})$ (octahedral) positions. These sextets are presented in Fig. 5 by red ( $\mathrm{Fe}(\mathrm{A})$ position) and blue $(\mathrm{Fe}(\mathrm{B})$ position) solid lines.

If compare the shapes of measured spectra for both samples the main differences are visible in the central part of spectra and relative intensities of sextets from (A) and (B) positions. In the case when $\mathrm{Ga}_{0.2} \mathrm{Fe}_{2.8} \mathrm{O}_{4}$ is the

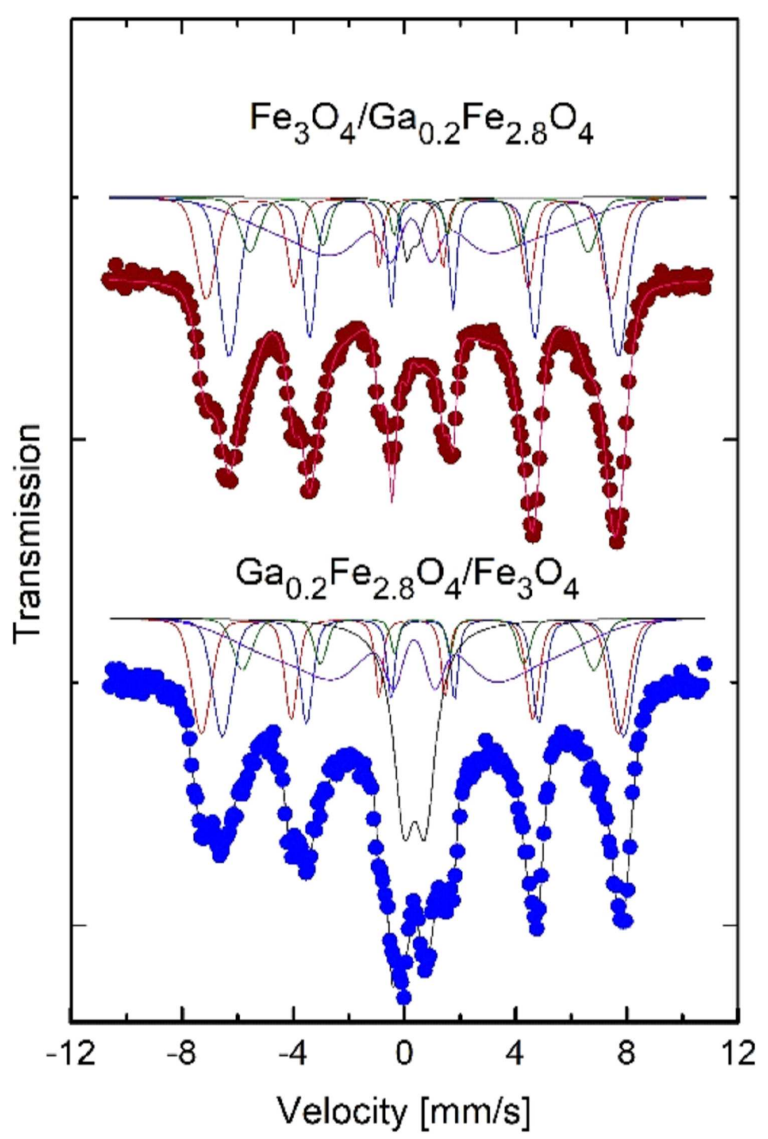

Fig. 5. RT Mössbauer spectra of core-shell nanoparticles. 
core the intensive doublet in the central part of spectra is visible and the intensity ratio of $I(\mathrm{~B}) / I(\mathrm{~A})=(1.1 \pm 0.2)$ is smaller than in the sample where $\mathrm{Ga}_{0.2} \mathrm{Fe}_{2.8} \mathrm{O}_{4}$ is as a shell where $I(\mathrm{~B}) / I(\mathrm{~A})=(1.6 \pm 0.2)$. Because in pure bulk magnetite $I(\mathrm{~B}) / I(\mathrm{~A})=2$ (assuming the Lamb factor $f(\mathrm{~A})=f(\mathrm{~B}))$ the simplest explanation of our results lead to the conclusion that $\mathrm{Ga}$ atoms are located preferentially in $\mathrm{B}$ position for sample when $\mathrm{Ga}_{0.2} \mathrm{Fe}_{2.8} \mathrm{O}_{4}$ is in core and almost evenly distributed in the case when $\mathrm{Ga}_{0.2} \mathrm{Fe}_{2.8} \mathrm{O}_{4}$ is in the shell. This is in contradiction with the conclusion obtained from our X-ray measurements and literature [7]. This simple analysis does not take into account the influence of preparation method on the properties of magnetite nanoparticles. Our earlier works showed that the "acac" preparation method, used in this work, for magnetite nanoparticles led to samples where the $I(\mathrm{~B}) / I(\mathrm{~A}) \approx 1[13,14]$ which means that the samples are so-called nonstoichiometric magnetite. Taking into account this experimental fact the increase of $I(\mathrm{~B}) / I(\mathrm{~A})$ between two our samples can be explained as a situation where the $\mathrm{Ga}$ atoms preferentially located in (A) sites when $\mathrm{Ga}_{0.2} \mathrm{Fe}_{2.8} \mathrm{O}_{4}$ is as the shell and evenly distributed in the sample where $\mathrm{Ga}_{0.2} \mathrm{Fe}_{2.8} \mathrm{O}_{4}$ is in the core. Such explanation supports the conclusion obtained from X-ray measurement and is more reliable. The observed doublet can be explained as superparamagnetic fluctuation of Fe magnetic moments. This means that $\mathrm{Ga}_{0.2} \mathrm{Fe}_{2.8} \mathrm{O}_{4}$ as a shell suppress superparamagnetic fluctuation of $\mathrm{Fe}$ moments in this samples.

As one can see the morphology of core-shell nanoparticles influence both on the magnetic properties and substitution of $\mathrm{Ga}$ atoms in the $\mathrm{A}$ and $\mathrm{B}$ positions in magnetite structure.

\section{Conclusions}

Inspection of the diffraction data leaves no doubt that (1) the homogeneity of the samples has been systematically achieved thanks to acac procedure of preparation and (b) Ga atoms preferentially substitute the A positions. SANS measurements confirmed the smaller than the other size of $\mathrm{Fe}_{3} \mathrm{O}_{4} / \mathrm{Ga}_{0.2} \mathrm{Fe}_{2.8} \mathrm{O}_{4}$ particles which leads to the much worse intensity to background ratio visible in the XRD data. The shape of particles is insensitive on the location of gallium admixture at magnetite matrix in contrast to the effective radius of nanoparticles including different ratios the cores to shells. MS data disclosed that $\mathrm{Ga}_{0.2} \mathrm{Fe}_{2.8} \mathrm{O}_{4}$ as a core suppress superparamagnetic fluctuation of Fe moments and fulfils the rule (b) but the morphology of core-shell nanoparticles influences both on the magnetic properties and in-site occupation of $\mathrm{Ga}$ atoms.

\section{Acknowledgments}

This work was partly sponsored by the Polish Government Plenipotentiary for JINR in Dubna (Project No. 04-4-1121-2015/2020) as well as has received financial support from the Polish Ministry of Science and Higher Education under subsidy for maintaining the research potential of the Faculty of Physics, University of Bialystok.

\section{References}

[1] S. Khizroev, M. Kryder, D. Litvinov, D. Thomson, Appl. Phys. Lett. 81, 2256 (2002).

[2] W. Brullot, N.K. Reddy, J. Wouters, V.K. Valev, B. Goderis, J. Vermant, T. Verbiest, J. Magn. Magn. Mater. 324, 1919 (2012).

[3] M. Banobre-Lopez, A. Teijeiro, J. Rivas, Rep. Pract. Oncol. Radiother. 18, 397 (2013).

[4] R.M. Cornell, U. Schwertmann, The Iron Oxides, VCH Press, Weinheim 1996.

[5] B. Kalska-Szostko, U. Wykowska, D. Satula, P. Nordblad, Beilstein J. Nanotechnol. 6, 1385 (2015).

[6] K. Rećko, J. Waliszewski, U. Klekotka, D. Soloviov, G. Ostapczuk, D. Satuła, M. Biernacka, M. Balasoiu, A. Basa, B. Kalska-Szostko, K. Szymański, Phase Transit. 91, 128 (2018).

[7] V.L. Pool, M.T. Klem, C.L. Chorney, E.A. Arenholz, Y.U. Idzerda, J. Appl. Phys. 109, 07B529 (2011).

[8] J. Rodriguez-Carvajal, Physica B 192, 55 (1993).

[9] A.I. Kuklin, A.D. Rogov, Yu.E. Gorshkova, P.K. Utrobin, Yu.S. Kovalev, A.V. Rogachev, O.I. Ivankov, S.A. Kutuzov, D.V. Soloviov, V.I. Gordeliy, Phys. Part. Nuclei Lett. 8, 119 (2011).

[10] A.I. Kuklin, D.V. Soloviov, A.V. Rogachev, P.K. Utrobin, Yu.S. Kovalev, M. Balasoiu, O.I. Ivankov, A.P. Sirotin, T.N. Murugova, T.B. Petukhova, Yu.E. Gorshkova, R.V. Erhan, S.A. Kutuzov, A.G. Soloviev, V.I. Gordeliy, J. Phys. Conf. Ser. 291012013 (2011).

[11] SAS-view, Small Angle Scattering Analysis Software Package.

[12] A. Guinier, G. Fournet, Small-Angle Scattering of X-ray, Wiley, New York 1995.

[13] U. Klekotka, B. Piotrowska, D. Satuła, B. KalskaSzostko, Appl. Surf. Sci. 444, 161 (2018).

[14] U. Klekotka, D. Satuła, S. Spassov, B. KalskaSzostko, Coll. Surf. A 537, 452 (2018). 